Article

\title{
Calculation of Target-Specific Point Distribution for 2D Mobile Laser Scanners
}

\author{
Conor Cahalane *, Conor P. McElhinney, Paul Lewis and Tim McCarthy \\ National Centre for Geocomputation, Iontas, NUI Maynooth, Maynooth, Co. Kildare, Ireland; \\ E-Mails: conormce@cs.nuim.ie (C.P.M.); paul.lewis@ nuim.ie (P.L.); \\ tim.mccarthy@nuim.ie (T.M.)
}

* Author to whom correspondence should be addressed; E-Mail: conor.cahalane@ nuim.ie;

Tel.: +353-1-708-6204; Fax: +353-1-708-6456.

Received: 13 March 2014; in revised form: 6 May 2014 / Accepted: 19 May 2014 /

Published: 27 May 2014

\begin{abstract}
The current generation of Mobile Mapping Systems (MMSs) capture high density spatial data in a short time-frame. The quantity of data is difficult to predict as there is no concrete understanding of the point density that different scanner configurations and hardware settings will exhibit for objects at specific distances. Obtaining the required point density impacts survey time, processing time, data storage and is also the underlying limit of automated algorithms. This paper details a novel method for calculating point and profile information for terrestrial MMSs which are required for any point density calculation. Through application of algorithms utilising 3D surface normals and 2D geometric formulae, the theoretically optimal profile spacing and point spacing are calculated on targets. Both of these elements are a major factor in calculating point density on arbitrary objects, such as road signs, poles or buildings-all important features in asset management surveys.
\end{abstract}

Keywords: mobile mapping systems; performance; LiDAR

\section{Introduction}

MMSs operating laser scanners are capable of producing high density point clouds, but this results in high data volumes and increased processing times. Manually interrogating this data is extremely time consuming and therefore automated algorithms play an important role in processing. These algorithms are designed to automatically recognise features in point clouds, thus eliminating or 
reducing the need for manual input. Figure 1a displays an example of three automated algorithms developed by [1], which have identified trees, poles and the road edge and other examples can be seen in work by [2-7]. The distribution of points on an object influences the success of the algorithm, for example [8-10] require a minimum number of points per scan line to recognise a cylindrical object and the work presented by [11] demonstrated the importance of a high point density on spatial accuracy.

Figure 1. Data processing and point distribution (a) automated algorithms identify features (b) vehicle velocity and MMS hardware configuration results in a corkscrew scanning pattern.

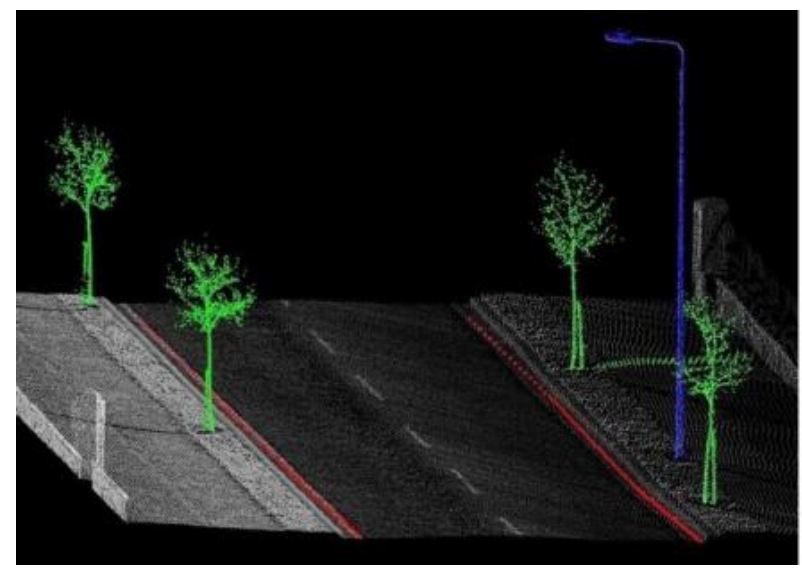

(a)

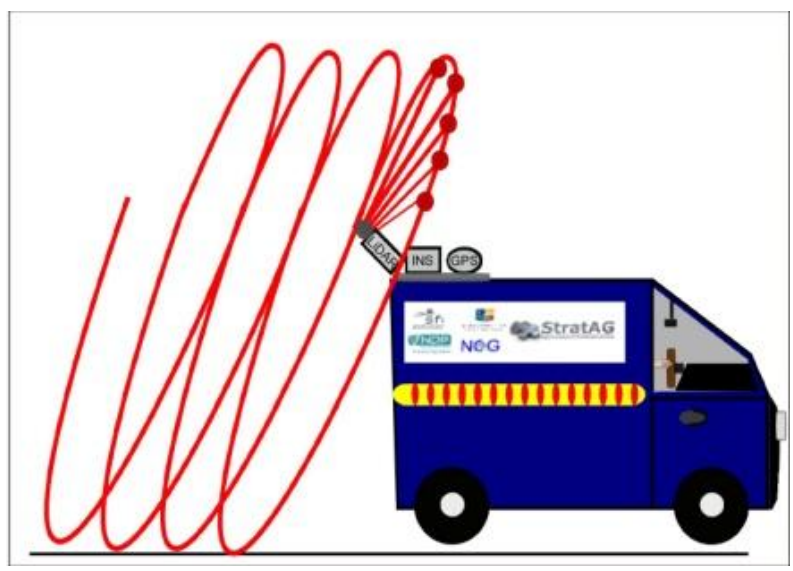

(b)

Defining point distribution is a complicated process. Hardware suppliers generally define scanner point distribution as the number of points per $\mathrm{m}^{2}$, yet for curved surfaces this confines a $3 \mathrm{D}$ value to a 2D measurement and ignores variations in point distribution across large targets. To include this third dimension, [12] projected all points per $\mathrm{m}^{3}$ onto a 2D circle. In [13] the authors also discussed point distribution and ways to define it for 3D data captured from multiple platforms, further reinforcing the link between point distribution and automated data processing. Part of the difficulty in defining point distribution is due to the fact that additional research is required to calculate what point distribution different platforms are capable of, particularly for project managers designing survey specifications. For example, one MMS survey specification [14] states that, 'the point density should be sufficient to identify and extract physical detail to the accuracy specified for the project' but does not specify what point density will permit that accuracy, or what MMS configuration will facilitate that point density. In another specification, [15] inform that clients requesting LiDAR surveys must carefully specify 'the point-cloud point density' but link this to the speed of vehicle only. In [16] this was improved significantly by subdividing point density into 'coarse', 'intermediate', and 'fine' and it was also recommended that a point density map with summary statistics be requested as a deliverable. It is our opinion that the distribution of the points on the surface is a possible compliment to the traditional point density definition. In [16] point distribution is explored, and the authors provide tabular information on what point spacing is required to achieve a specific point density, but do not define what MMS hardware, hardware configuration or operating parameters are required to achieve that point spacing.

At present there are no generic, robust methods for quantifying point distribution and we believe the procedure set down in this paper will be the first example of this. Modern MMSs operate a 2D, 
full-circle laser scanner designed for mobile surveys. 2D scanners utilise the forward motion of the vehicle to provide 3D data (Figure $1 \mathrm{~b}$ ). When this scanning pattern intersects with a planar surface, the laser points are distributed over the surface in a linear pattern. These lines are termed, 'scan profiles'. The gaps formed between each scan profile is the 'profile spacing' (Figure 2a). The angle of the scan profile on the target is influenced by the orientation of both the scanner and the target. This angle is termed the 'profile angle' and is illustrated in Figure $2 \mathrm{~b}$. The vehicle speed, the orientation of the scanner(s), the scanner mirror frequency $\left(\mathrm{M}_{\mathrm{f}}\right)$ and the orientation of the object all influence scan profiles. The distance between subsequent points along a scan profile is known as the 'point spacing', as illustrated in Figure 2c. Point spacing is influenced by a number of factors, including: the pulse repetition rate (PRR), which is the number of pulses per second, $\mathrm{M}_{\mathrm{f}}$, which controls the number of mirror rotations per second, the range to the target from the scanner, the scanner's field of view (FOV), the height difference between target and scanner, the orientation of the target and finally the orientation of the scanner. The orientation of the scanner is important for maximising coverage of the environment and can be varied between surveys. Scan hardware settings are also variable and dependant on the smallest target in the survey specification. Accurately calculating the influence that any variation in configuration or settings has on point distribution is important for optimising MMS performance. This paper presents a novel and accurate method to do this.

Figure 2. A corkscrew scan pattern intersecting with a vertical surface results in three distinctive features (a) profile spacing (b) profile angle and (c) the point spacing.

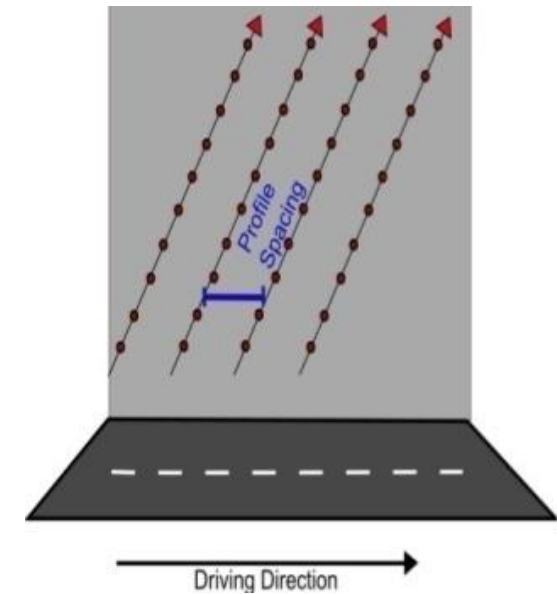

(a)

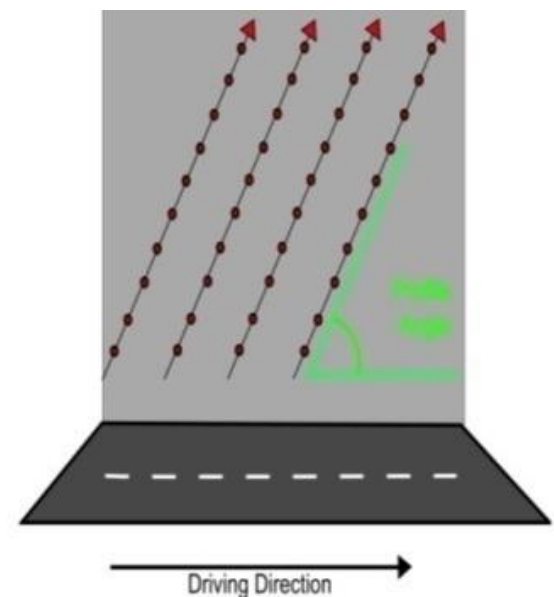

(b)

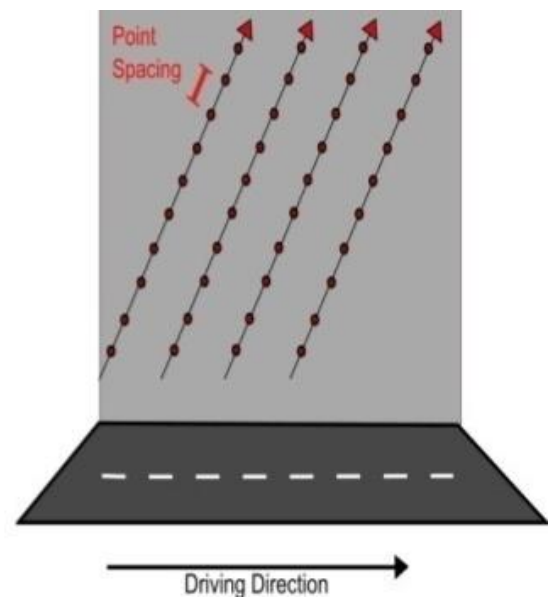

(c)

Point distribution is not only of interest to MMSs. In [17] the resolution of terrestrial laser scanner (TLS) point clouds was investigated and [18,19] have investigated the smallest feature that can be recognised in a TLS point cloud. Research like this is of benefit to TLS operators surveying fine-detail like statues or paintings, but the majority of MMSs are unsuitable for these surveys. Although it is possible to mount a TLS on a vehicle and use it in 'stop and go' mode, these are not suitable for mobile surveys. In 'stop and go' a tripod mounted scanner is placed on a moving platform and driven to a survey point [20]. The vehicle stops at this point and then scans the entire scene. The vehicle then moves to the next survey point and this process is repeated. To operate a TLS on a moving platform, the TLS must have one of its axes of rotation locked so it can operate in 'profile mode' [21,22]. However, due to their popularity with MMS designers, e.g., the Optech Lynx [23], Trimble MX8 [24] 
and StreetMapper [25], the point and profile algorithms described in this paper focus on 2D full circle scanners only. Existing work in this area calculates point distribution through three main methods: manual measurements, geometric formulae and LiDAR simulations.

\subsection{Manual Measurements}

By locking one rotation axis of a FARO TLS, [22] operated in profile mode and then manually measured the profile and point spacing at different ranges, speeds and scan frequencies post-mission. It was then possible to approximate what point distribution a user could expect from that MMS at a similar survey site. In [20] the authors operated a Leica HDS in profile mode and provided a table listing profile spacing at three vehicle speeds and three $\mathbf{M}_{\mathrm{f}}$. Only [22] incorporated scanner rotations. Unlike TLSs such as the Leica HDS 4500, vertical scanner rotations can be implemented with the FARO. Neither study incorporated dual-axis scanner rotations (a horizontal and a vertical rotation) or provided a method to calculate point distribution.

\subsection{Geometric Formulae}

Most laser scanner manufacturers provide information on the point density that a user can expect from their hardware. Riegl [26] provide detailed graphs plotting the point density a user can expect for various scanner, target and vehicle parameters. This is useful in the mission planning stage, however, it does not incorporate scanner rotations, target rotations or any height difference between scanner and target. These graphs are also hardware specific. These graphs are limited to specifying point density by calculating point spacing at a single target location, whereas point spacing varies over a target, something that is particularly relevant for angled surfaces and in [27] we discussed the significance of this. RiACQUIRE [28] is a useful mission planning tool that can calculate profile spacing, point pacing and point density for Riegl scanners on a target, however, RiACQUIRE does not incorporate horizontal or vertical scanner rotations, horizontal or vertical target rotations, height difference between scanner and target or different target types. Additional geometric formulae have been employed by [29] to identify the effect that vehicle direction and velocity has on scan profiles. Their work focused on automatic detection of objects and the purpose of this calculation was to eliminate areas of low point density to decrease processing time. In [30] useful formulae and graphical information are provided for calculating point spacing and point density on the road surface but limited the inputs to their formulae to the PRR, $\mathrm{M}_{\mathrm{f}}$, measurement range and vehicle velocity.

\subsection{Simulations}

A LiDAR simulation models the real world interaction between a LiDAR system and the terrain. They have been used for investigating aerial platforms [31], but the viewing geometry is less complicated for aerial platforms and the FOV is more restricted. The authors of [32] designed and tested a simulator for assessing errors, algorithm development and system validation for aerial and terrestrial systems. Simulators are useful tools for assessing point clouds or for algorithm development, but do not provide a method for calculating point distribution. Although [12] included important elements in their simulator: dual-axis scanner rotations, variations in scanner location, different PRRs 
and different $\mathrm{M}_{\mathrm{f}}$, their simulator did not calculate point distribution. Once any simulation is complete, point and profile measurements are manual and localised to a single point on a target or an average measurement is applied to the area in question and a colour scheme is applied to aid with visualisation.

\section{Calculating Point and Profile Information}

In [27] we introduced the overall system, the MobIle Mapping poInt density Calculator, or MIMIC. MIMIC calculates the theoretically optimal position that each laser pulse strikes a surface at using geometric formulae. Random events such as multiple returns, occlusions or changes in the width of the laser footprint due to an increase in scan range or the orientation of the surface are not modelled in MIMIC. Although these factors may change the position or number of individual laser pulses, the purpose of MIMIC is to provide a tool for assessing MMS hardware configurations during the mission planning stage or during MMS benchmarking and therefore a standard scenario (the theoretically optimal position of each pulse) is employed. In the following sections the modules for calculating point distribution are detailed.

\subsection{Profile Angle Module}

Changes in profile angle $\left(\theta_{\operatorname{PrA}}\right)$ were first explored and quantified in [33]. A method to calculate $\theta_{\operatorname{PrA}}$ was then detailed and validated for dual-axis scanner rotations and dual-axis target rotations in [34]. $\theta_{\operatorname{PrA}}$ was calculated through a combination of rotation matrices, 3D surface normals and 2D geometry. For example, in:

$$
\text { Rotation Matrix }\left(\mathrm{S}_{\text {rotated }}\right)=\mathrm{R}_{\mathrm{x}}\left(\gamma_{\text {scan }}\right) \mathrm{R}_{\mathrm{y}}\left(\beta_{\text {scan }}\right) \mathrm{R}_{\mathrm{z}}\left(\alpha_{\text {scan }}\right)
$$

$\gamma, \beta$ and $\alpha$ are the vertical, axial and horizontal rotation angles of the scanner. Figure 3 illustrates and Table 1 lists the terminology for the different rotations applied in this paper for both scanner and target. In [34] we showed that this method was susceptible to errors in MMS calibration and also to vehicle dynamics such as pitch, roll and yaw but that accuracy was high and suitable for MMS assessment.

Figure 3. Horizontal and vertical axes of rotation for both target and scanner (a) MMS and target (b) target axes of rotation (c) scanner axes of rotation.

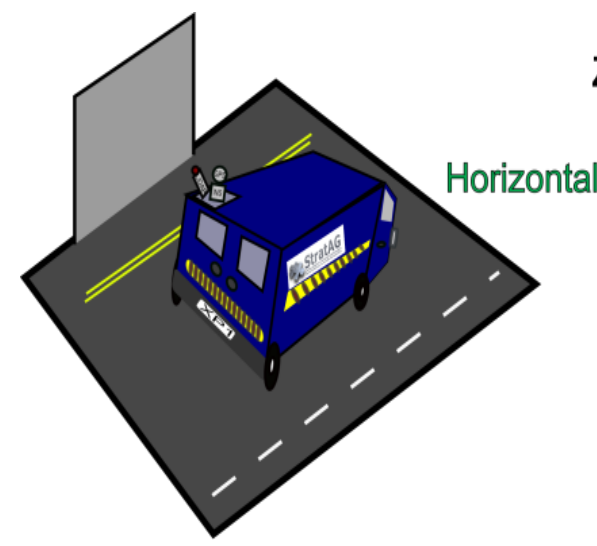

(a)

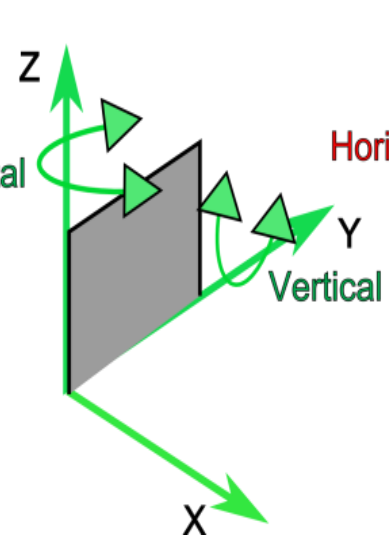

(b)

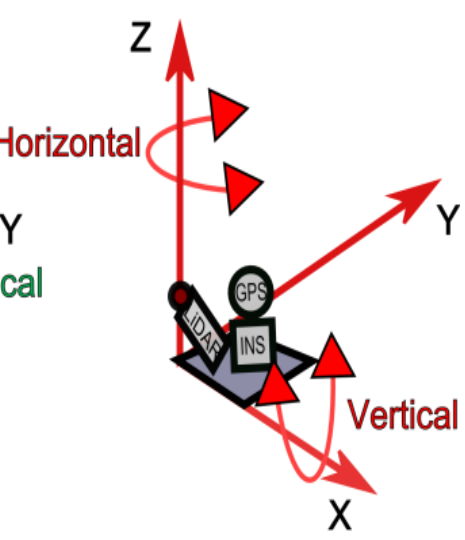

(c) 
Table 1. Terminology for horizontal and vertical scanner and target rotations.

\begin{tabular}{ccc}
\hline Rotation Axis & Target & Scanner \\
\hline Horizontal & $\alpha_{\mathrm{targ}}$ & $\alpha_{\text {scan }}$ \\
Vertical & $\beta_{\mathrm{targ}}$ & $\gamma_{\text {scan }}$ \\
\hline
\end{tabular}

\subsection{Calculating Profile Spacing}

Although a basic method for calculating profile spacing for dual axis scanner rotations on the road surface was detailed and validated in [35], a more advanced method is required for parallel vertical surfaces or those that have been inclined or rotated (these are referred to as 'angled' in this paper). Figure 4a shows a vertical surface with scan profiles striking it. Depending on whether a target is angled towards or away from the MMS, the distance between these scan profiles will decrease or increase. The distance, $\mathrm{d}$ is the distance travelled by the vehicle in one mirror rotation and can also be referred to as the horizontal profile spacing, $\mathrm{d}_{\mathrm{PrSH}}$. For a parallel, vertical target $\mathrm{d}$ and $\mathrm{d}_{\mathrm{PrSH}}$ are identical, but for angled targets they differ. For these formulae, $d_{P r S H}$ is referred to as $d+$ and $d-$ to illustrate the difference between the direction of rotation of the target. The vertical profile spacing, $\mathrm{d}_{\mathrm{PrSV}}$ is the vertical distance between scan profiles and is also illustrated in Figure 4a. Calculating profile spacing on an angled surface rotated away from the MMS, d+ in Figure 4b, requires:

$$
d+=\frac{d * \sin \theta_{3}}{\sin \theta_{4}}
$$

and the profile spacing on an angled surface rotated towards the MMS, d- in Figure 4c, requires:

$$
d-=\frac{d * \sin \theta_{2}}{\sin \theta_{5}}
$$

To calculate $d_{P r S v}$, the profile angle, $\theta_{\operatorname{PrA}}$ and the horizontal profile spacing, $d_{P r S H}$ are required. $d_{P r S V}$ can be calculated using:

$$
d_{\mathrm{PrSV}}=\frac{d_{\mathrm{PrSH}} * \sin \theta_{\mathrm{PrA}}}{\cos \left(\theta_{\mathrm{PrA}}\right)}
$$

Figure 4. Calculating profile spacing (a) profile spacing on a parallel vertical surface (b) aerial view of surface rotated away from the MMS-profile spacing increases (c) target is rotated towards the MMS-profile spacing decreases.

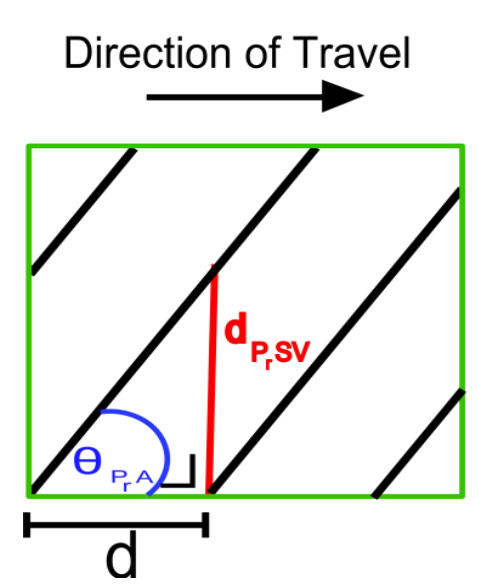

(a)

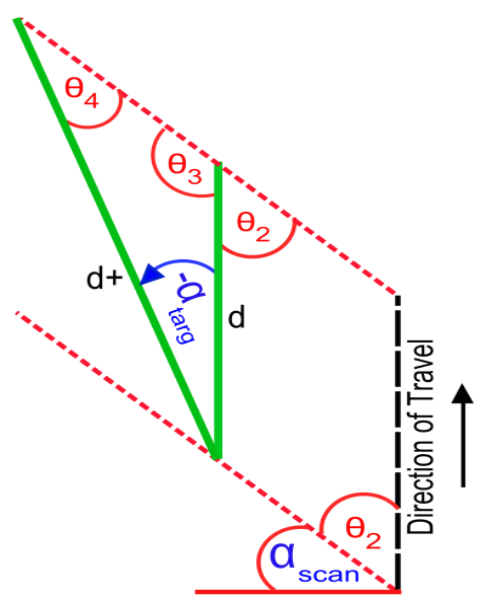

(b)

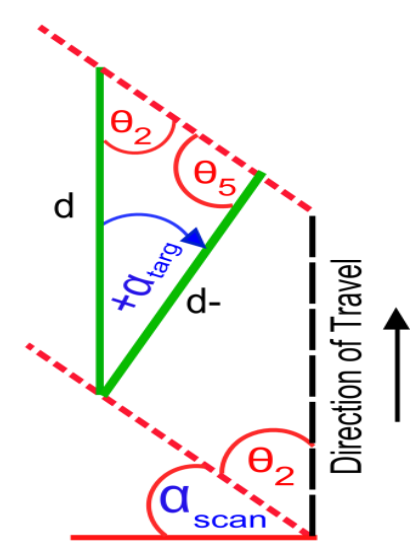

(c) 


\subsection{Calculating Point Spacing}

A method for calculating the point spacing, $\mathrm{d}_{\mathrm{PS}}$, for the road surface was detailed in [33]. These tests identified that the uncertainty in the road gradient introduced errors into a point spacing calculation that was carried out using planar targets. A novel point spacing calculation method for angled targets is applied in this paper. The first step in computing $d_{P S}$ is calculating the angular step width of the scanner, $\theta_{\mathrm{A}}$. To find $\theta_{\mathrm{A}}$, the number of points per mirror rotation, $\mathrm{P}_{\mathrm{p}} \mathrm{M}$, must be identified. Following this, $\theta_{\mathrm{A}}$ can be found using the FOV of the scanner and the $\mathrm{P}_{\mathrm{p}} \mathrm{M}$ :

$$
\theta_{\mathrm{A}}=\frac{\mathrm{FOV}}{\mathrm{PpM}}
$$

MIMIC calculates $d_{P S}$ through the application of $2 \mathrm{D}$ geometric formulae to calculate the distance between subsequent laser pulses on the angled surface, however a horizontal and vertical rotation of the scanner alters the orientation of the scan plane in relation to the target and the scanner. This alters the viewing geometry for the $2 \mathrm{D}$ plane and therefore the height of the scanner and the target must be adjusted accordingly in the calculation. $Z_{\text {scan }}$ and $Z_{\text {targ }}$ represent these adjusted heights in Figure 5a. The amended heights can be calculated using the original target and scanner heights $\left(\mathrm{h}_{\text {targ }}, \mathrm{h}_{\text {scan }}\right)$ and the vertical scanner rotation, $\beta_{\text {targ }}$, with:

$$
Z_{\mathrm{targ}}=\frac{h_{\text {targ }}}{\cos \left(\gamma_{\mathrm{scan}}\right)}
$$

and:

$$
Z_{\text {scan }}=\frac{h_{\text {scan }}}{\cos \left(\gamma_{\text {scan }}\right)}
$$

The height difference between the scanner and the target, $\left(Z_{\text {diff }}\right)$, is required for these calculations on the $2 \mathrm{D}$ plane. The horizontal range to the target, $\mathrm{H}_{\mathrm{r}}$, must be specified by the user. For this part of the calculation, the target is assumed to be a hypothetical point at the same height as the scanner. Therefore the range from the scanner to the target is $\mathrm{r} 1$ in Figure 5a. $\mathrm{r} 1$ is dependent on the scanner horizontal rotation, $\alpha_{\text {scan }}$ and $\mathrm{H}_{\mathrm{r}}$. $\mathrm{r} 1$ can be calculated with:

$$
r 1=\frac{H_{r}}{\cos \left(\alpha_{\text {scan }}\right)}
$$

Figure 5. Calculating point spacing (a) MIMIC requires an adjusted height of scanner and height of target (b) adjusted for vertical rotation of target.

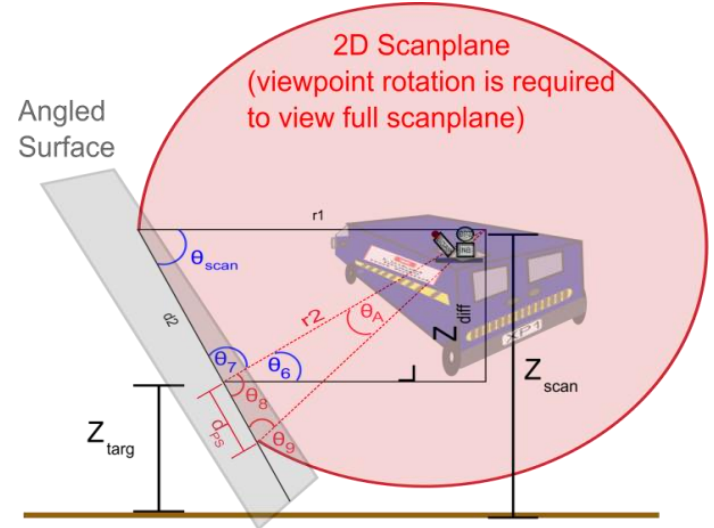

(a)

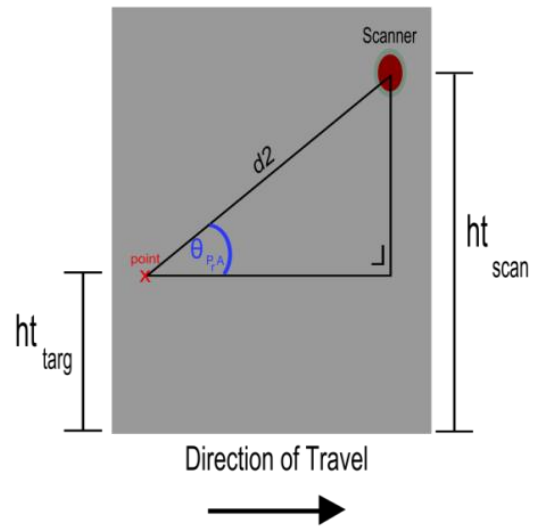

(b) 
Additional variables are required for calculating $d_{P S}$. The next value that is required is the scan angle, $\theta_{\text {scan. }}$. This is the angle formed between $\mathrm{r} 1$ and the scan profile on the angled surface, $\mathrm{d} 2$. This is calculated in a similar manner to the profile angle as detailed in [34].

Unlike existing methods, MIMIC can incorporate different target heights. Once MIMIC has calculated $\theta_{\mathrm{A}}, \mathrm{r} 1, \theta_{\text {scan }}$ and $\mathrm{Z}_{\mathrm{diff}}$, it can calculate $\mathrm{r} 2$. Unlike the horizontal range, $\mathrm{H}_{\mathrm{r}}$, or the hypothetical range at $\mathrm{r} 1, \mathrm{r} 2$ is the actual range to the target along the scan plane. $\mathrm{d} 2$ is required to calculate $\mathrm{r} 2$ and is the portion of the scan plane that has intersected with the angled plane between the hypothetical point at $\mathrm{r} 1$ and the actual point at $\mathrm{h}_{\text {targ. }}$. Calculating $\mathrm{d} 2$ does not require the same rotated perspective that the previous calculation did, but rather one that was adjusted for the vertical rotation of the target only. Therefore $\mathrm{ht}_{\text {scan }}$ and $\mathrm{ht}_{\mathrm{targ}}$ are used. Figure $5 \mathrm{~b}$ illustrates these variables. Once $\mathrm{d} 2$ has been calculated the actual range to the target $(\mathrm{r} 2)$ can be calculated with:

$$
r 2=\sqrt{d 2^{2}+r 1^{2}-2\left((d 2 * r 1)\left(\cos \left(\theta_{\text {scan }}\right)\right)\right.}
$$

Once $\mathrm{r} 2$ has been calculated, the point spacing, $\mathrm{d}_{\mathrm{PS}}$, can then be found using:

$$
d_{\mathrm{PS}}=\frac{(r 1)\left(\sin \theta_{\mathrm{A}}\right)}{\left(\sin \theta_{9}\right)}
$$

\section{Experimental Datasets}

Two scenarios involving three datasets were analysed to verify the calculations. A constructed 3D model was used as the control dataset as all external and hardware errors could be eliminated, and point clouds from the MMS designed at the NCG, the XP1, and from a commercial MMS, the Optech Lynx M1 [23], were used to experimentally validate MIMIC's calculations with real-world data.

\subsection{Dataset 1-Constructed CAD Models}

For our initial tests, we created a number of planes representing different surfaces and different scanner rotations in Bentley Microstation v8i. Here we measured the interaction between lines, planes and discs. Planes represented targets, discs represented the scan plane and lines represented individual laser pulses. Hardware or configuration issues were therefore absent from these tests. Additionally, external forces such as pitch, roll or yaw of the MMS could not influence the point and profile measurements. Dataset 1 consisted of 14 targets, including horizontal and vertical surface rotations for a dual axis scanner rotation (Figure 6a). We then manually measured the profile spacing and point spacing on each surface for each dual axis scanner rotation in the CAD environment.

\subsection{Point Cloud Data}

Two MMSs were used to validate MIMIC's calculations. The first was the research platform designed by the team at the NCG and the second was using data supplied by Optech Inc. (ON, Canada) from their commercial system, the Optech Lynx M1. 
Figure 6. Validation (a) initial tests with constructed CAD models (b) survey data captured by the XP1 (c) survey data captured by the Optech Lynx M1.

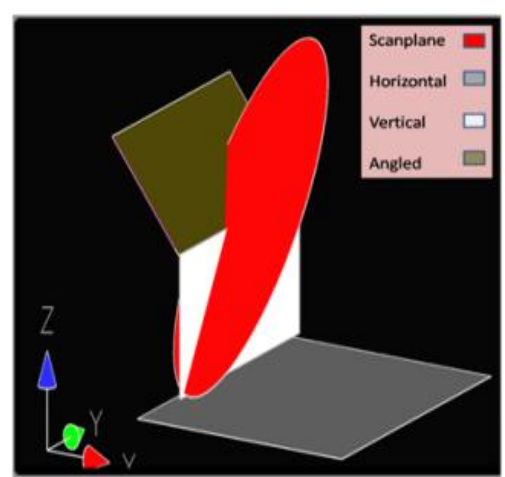

(a)

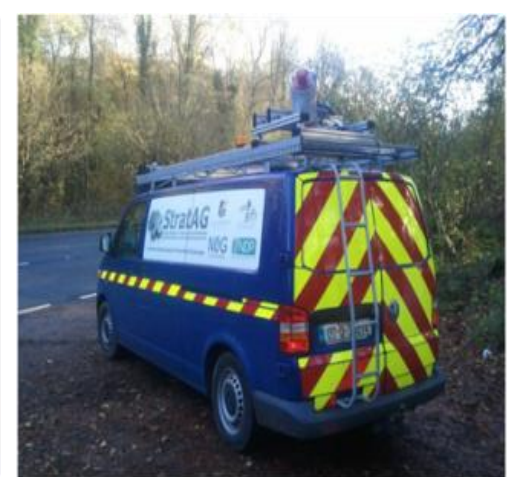

(b)

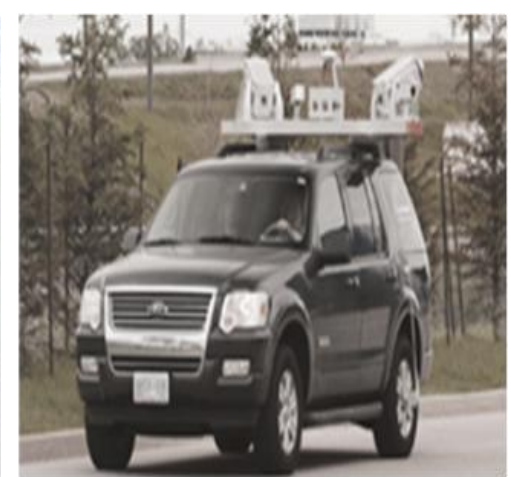

(c)

\subsubsection{Dataset 2-XP1 MMS}

The multi-disciplinary research group StratAG, established to research advanced geotechnologies at NUI Maynooth have designed and developed a multi-purpose land based Mobile Mapping System, the XP1. The primary components of the XP1 (Figure 6b) are an IXSEA LANDINS (Paris, France), a Riegl VQ-250 $300 \mathrm{kHz}$ (Horn, Austria) laser scanner and an imaging system consisting of six progressive-scan cameras. Imaging sensors include a thermal camera and a multi-spectral camera capable of sensing across blue, green, red and two infra-red bandwidths. Unlike most commercial systems, the XP1 is a single scanner system.

\subsubsection{Dataset 3-Optech Lynx}

The commercial system was the Optech Lynx M1 and it provided the opportunity for further validation with different scan hardware and a different system configuration. Unlike the XP1, the Optech Lynx M1 (Figure 6c), is a dual scanner MMS. Each 2D scanner is capable of a $500 \mathrm{kHz}$ PRR and a $200 \mathrm{~Hz} \mathrm{M}_{\mathrm{f}}$. Data from the Optech Lynx data facilitated system verification in a number of ways. Firstly, errors in calibration of the scanners on each MMS could lead to problems measuring target orientations. As it was not possible to verify the orientation of the surfaces using traditional survey methods, the use of two MMS improved the robustness of our tests. Secondly, the dual scanner Optech Lynx was used to verify that MIMIC can cater for dual scanner MMSs as the variations in scanner configuration between the Optech Lynx and the XP1 further verified MIMIC's profile calculations for different system configurations. Finally, the Optech M1 scanner is capable of operating at a higher $\mathrm{M}_{\mathrm{f}}$ than the Riegl VQ-250 onboard the XP1, providing further test data.

\subsubsection{Target Selection and Validation Procedure}

For both MMS datasets a number of suitable areas for tests were selected and a series of sample measurements were recorded at each location. Using software developed by researchers at the NCG [36-38], areas consisting of suitable man-made vertical structures (e.g., walls, buildings, roofs, road-side infrastructure) were quickly identified and XP1 survey data was extracted from large files of both rural and urban environments in Ireland. Optech Inc. supplied researchers at the NCG with survey 
data captured in the vicinity of their offices in Ontario. A set of 16 surfaces were then manually selected from the XP1 and Optech Lynx datasets because by ensuring variation in orientation, range and elevation of the target our calculations could be robustly validated. Each surface had a different elevation in relation to the MMS ( $\pm 2 \mathrm{~m}$ ) and was located at between $5 \mathrm{~m}$ and $16 \mathrm{~m}$ horizontal range to the scanner. Point density varied per target from 300 points to 1,500 points per $\mathrm{m}^{2}$. The orientation of each surface varied in horizontal from $-60^{\circ}$ to $+60^{\circ}$ and in vertical from $0^{\circ}$ to $45^{\circ}$. The range to and orientation of each surface was measured in the point cloud using measuring tools in Bentley Microstation v8i and TerraScan [39]. The inertial measurement units (IMUs) onboard each of the MMSs provided high accuracy velocity and orientation information making it possible to choose scan profiles captured at constant vehicle speeds for profile spacing tests and when vehicle dynamics (i.e., pitch, roll) were minimal. Profile spacing and point spacing were manually measured from the point cloud. The potential for error arose when manual approximation of the scan profile was inhibited by non-uniform point distribution along the scan profile. To assess the impact of this error, five profile spacing measurements were recorded from a single survey for two scan profiles, Profile 1 and Profile 2. Profile 1 displayed non-uniform point distribution while Profile 2 displayed a uniform point distribution. The standard deviation of these measurements $(\sigma)$ was then calculated. As expected, the profile exhibiting the highest $\sigma$ (implying a degree of interpretation when delineating a profile) also exhibited the higher errors, $0.003 \mathrm{~m}$ as opposed to $0.001 \mathrm{~m}$ as summarised in Table 2.

Table 2. Profile spacing tests- assessing the reliability of manual profile measurements as a validation tool for MIMIC-the importance of accurately delineating profiles in the point cloud is demonstrated by the positive correlation between $\sigma$ and error.

\begin{tabular}{ccc}
\hline Result & Profile 1 $(\mathbf{m})$ & Profile 2 $(\mathbf{m})$ \\
\hline Measured (Average) & 0.109 & 0.096 \\
MIMIC & 0.112 & 0.095 \\
$\sigma$ & 0.008 & 0.001 \\
Error & 0.003 & 0.001 \\
\hline
\end{tabular}

\section{Results and Discussion}

This section details the validation methodology and the results of the profile and point spacing tests. The tests using the constructed models are presented separately to the point cloud tests.

\subsection{Profile Spacing}

To ensure robust validation, MIMIC's profile spacing calculations were first theoretically validated using the constructed CAD models and then using point cloud data from the XP1 and the Optech MMS.

\subsubsection{Constructed Models}

Vertical surfaces rotated horizontally and vertically around the $\mathrm{Y}$ and $\mathrm{Z}$ axes respectively were used to validate MIMIC's profile spacing calculations for angled planes. In these initial tests the surface was rotated both clockwise and anti-clockwise around the $\mathrm{Z}$ axis. This represented a horizontal rotation of the target in either direction. The same process was applied for rotations around the $\mathrm{Y}$ axis which 
represented inclinations of the target. Combinations of horizontal and vertical rotations were also applied. For these tests a constant vehicle velocity of $10 \mathrm{~m} / \mathrm{s}$ was applied and a horizontal/vertical dual-axis scanner rotation referred to as $45^{\circ} / 45^{\circ}$ was applied. The initial profile spacing measurements return a zero error (Table 3), theoretically validating MIMIC's calculations of profile spacing for angled surfaces with a controlled dataset.

Table 3. Profile spacing-The profile spacing calculated by MIMIC validated using manual measurements on a constructed CAD model for a selection of horizontally rotated $\left(\alpha_{\text {targ }}\right)$ and vertically rotated $\left(\beta_{\text {targ }}\right)$ surfaces.

\begin{tabular}{cccccc}
\hline No. & $\boldsymbol{\alpha}_{\text {targ }}$ & $\boldsymbol{\beta}_{\text {targ }}$ & MIMIC (m) & CAD (m) & Error (m) \\
\hline 1 & $15^{\circ}$ & $0^{\circ}$ & 0.082 & 0.082 & 0.000 \\
2 & $0^{\circ}$ & $15^{\circ}$ & 0.100 & 0.100 & 0.000 \\
3 & $15^{\circ}$ & $15^{\circ}$ & 0.082 & 0.082 & 0.000 \\
4 & $-15^{\circ}$ & $0^{\circ}$ & 0.141 & 0.141 & 0.000 \\
5 & $0^{\circ}$ & $-15^{\circ}$ & 0.100 & 0.100 & 0.000 \\
\hline
\end{tabular}

Figure 7. MIMIC's profile spacing calculations: Calculating average error over five measurements plotted against measurement reliability $(\sigma)$ for horizontal and vertical profile spacing at two mirror frequencies $-100 \mathrm{~Hz}$ and $150 \mathrm{~Hz}$.

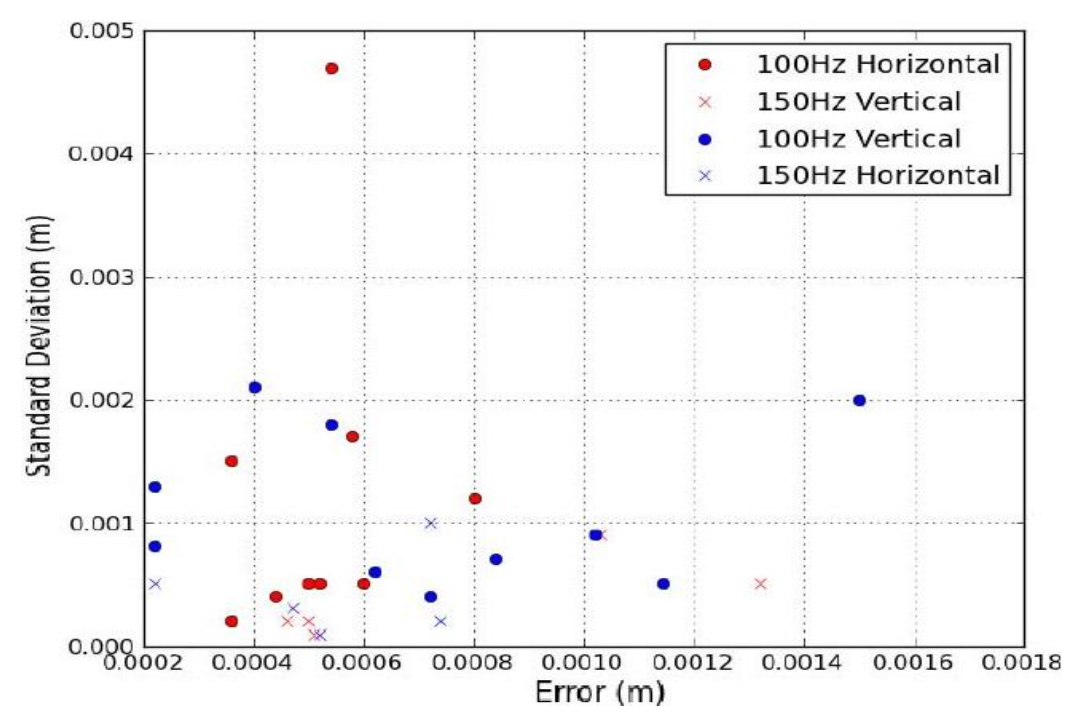

\subsubsection{Point Cloud Data}

Angled structures were selected from the XP1 and Optech Lynx datasets. This enabled validation of MIMIC using point cloud data captured at different vehicle velocities. The Optech Lynx dataset enabled verification of a second scanner configuration and a higher $\mathrm{M}_{\mathrm{f}}$ than that from the XP1 alone. Data was chosen at two $\mathrm{M}_{\mathrm{f}}, 100 \mathrm{~Hz}$ and $150 \mathrm{~Hz}$ and vehicle velocity varied for each target. Targets 1-6 were surfaces exhibiting pronounced vertical rotations whereas Targets $7-15$ focussed on horizontal surface rotations. Five measurements were recorded for both $d_{\mathrm{PrSH}}$ and $\mathrm{d}_{\mathrm{PrSV}}$ for each set of scan profiles. $\sigma$ was then calculated to provide an indication of the quality of each measurement. The errors from the point cloud tests were higher than the control dataset but were still low, less than $1 \mathrm{~mm}$ in all 
but four of the cases. Figure 7 illustrates the errors and plots them against $\sigma$ for both horizontal and vertical profile spacing. This helps visualise the quality of the measurements used to validate the calculated values. It can be seen that the majority of MIMIC's calculations have an error of less than $1 \mathrm{~mm}$ and a $\sigma$ of less than $2 \mathrm{~mm}$. The low $\sigma$ implies that the measurements were reliable, and the low error validates experimentally MIMIC's calculations for profile spacing on angled surfaces at different scanner configurations and vehicle velocities.

\subsection{Point Spacing}

MIMIC's point spacing calculations were also validated using the constructed CAD model as a control dataset and then using the XP1 and Optech point cloud data to assess their performance with real world data.

\subsubsection{Constructed Models}

Horizontal and vertical target rotations were introduced to verify MIMIC's point spacing calculations for an angled surface. The range from the scanner to the target was fixed but the target orientation was varied. For the initial CAD tests a vertical surface was placed in a selection of orientations and a series of 3D lines representing individual laser pulses were created. The hypothetical scanner was fixed at an elevation of $3.1 \mathrm{~m}$, orientated at $45^{\circ} / 45^{\circ}$ and placed at a range of $4 \mathrm{~m}$ from the target, although the range varied slightly depending on the rotation of the target. A $\theta_{\mathrm{A}}$ of $0.12^{\circ}$ was applied. The target was rotated in $15^{\circ}$ steps horizontally, vertically and a dual axis rotation was also applied. Anti-clockwise and clockwise rotations were introduced. The 3D lines were intersected with the angled surface and the distance between the two points of intersection was measured. The results are displayed in Table 4. The control tests theoretically validate MIMIC's method for calculating point spacing on angled surfaces, returning a zero error.

Table 4. Point spacing-The point spacing calculated by MIMIC validated using manual measurements from a constructed CAD model for a selection of horizontally rotated $\left(\alpha_{\text {targ }}\right)$ and vertically rotated $\left(\beta_{\text {targ }}\right)$ surfaces. The simulated scanner height was $3.1 \mathrm{~m}$, and a $\theta_{\mathrm{A}}$ of $0.12^{\circ}$ was applied.

\begin{tabular}{cccccc}
\hline No. & $\boldsymbol{\alpha}_{\text {targ }}$ & $\boldsymbol{\beta}_{\text {targ }}$ & MIMIC $(\mathbf{m})$ & CAD $(\mathbf{m})$ & Error $(\mathbf{m})$ \\
\hline 1 & $15^{\circ}$ & $0^{\circ}$ & 0.013 & 0.013 & 0.000 \\
2 & $0^{\circ}$ & $15^{\circ}$ & 0.020 & 0.020 & 0.000 \\
3 & $15^{\circ}$ & $15^{\circ}$ & 0.016 & 0.016 & 0.000 \\
4 & $30^{\circ}$ & $0^{\circ}$ & 0.010 & 0.010 & 0.000 \\
5 & $0^{\circ}$ & $30^{\circ}$ & 0.028 & 0.028 & 0.000 \\
6 & $30^{\circ}$ & $30^{\circ}$ & 0.021 & 0.021 & 0.000 \\
7 & $-30^{\circ}$ & $0^{\circ}$ & 0.034 & 0.034 & 0.000 \\
8 & $0^{\circ}$ & $-30^{\circ}$ & 0.006 & 0.006 & 0.000 \\
9 & $-30^{\circ}$ & $-30^{\circ}$ & 0.006 & 0.006 & 0.000 \\
\hline
\end{tabular}




\subsubsection{Point Cloud Data}

The distance between subsequent points on the angled surface was measured manually for real-world point cloud data, so this once again introduced a potential error. Point spacing was measured five times on 16 targets and $\sigma$ calculated to provide an estimate of the quality of the 80 measurements. Targets $1-6$ were surveyed using the XP1's Riegl VQ-250 operating at a $300 \mathrm{kHz}$ PRR with a $\mathrm{M}_{\mathrm{f}}$ of $100 \mathrm{~Hz}$ and Targets 7-11 by the Optech Lynx operating at a $125 \mathrm{kHz}$ PRR and with a $\mathrm{M}_{\mathrm{f}}$ of $100 \mathrm{~Hz}$. Targets 12-16 were surveyed using the same scanner operating at a $500 \mathrm{kHz}$ PRR and a $\mathrm{M}_{\mathrm{f}}$ of $200 \mathrm{~Hz}$. By altering the target rotation for each test, the range to the target, the height difference between the scanner and target, the PRR and the $\mathrm{M}_{\mathrm{f}}$, MIMIC's capabilities of predicting point spacing were tested robustly. Figure 8 illustrates the error plotted against $\sigma$. The highest $\sigma$ was approximately $2 \mathrm{~mm}$ however, the majority of the measurements displayed less than $1 \mathrm{~mm}$. The largest error was less than $2 \mathrm{~mm}$ at a range of approximately $8 \mathrm{~m}$. These results validate MIMIC's calculations using real-world MMS datasets.

Figure 8. MIMIC's point spacing calculations: Calculating average error over five point spacing measurements plotted against measurement reliablity $(\sigma)$ for three PRRs and two $\mathrm{M}_{\mathrm{f}} \mathrm{s}$.

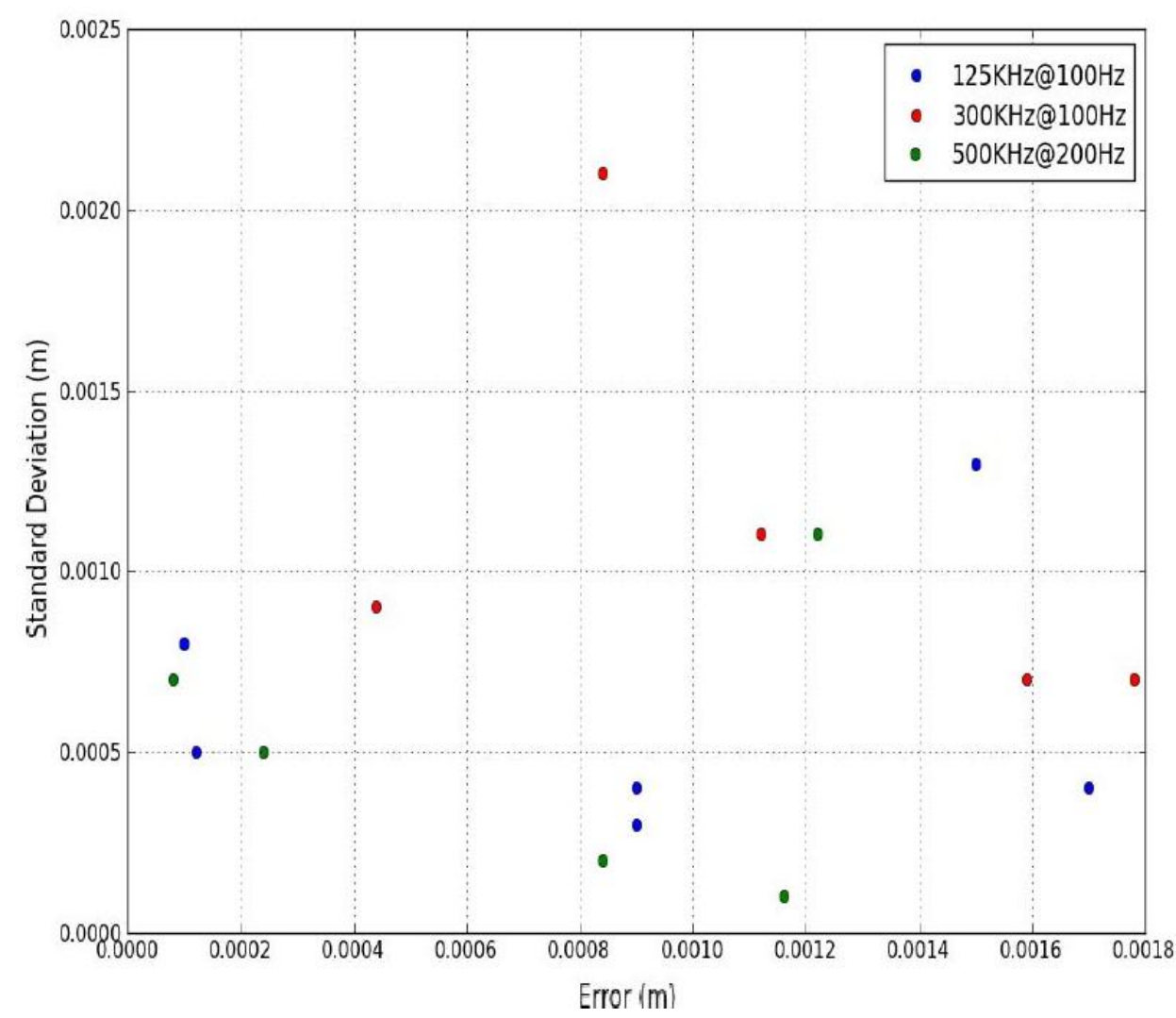

\subsection{Discussion}

Table 5 summarises the errors for each of the tests. The largest error is $2 \mathrm{~mm}$, whereas the maximum mean error is $1 \mathrm{~mm}$. This is satisfactory, but for large targets with a low profile spacing an error of $1 \mathrm{~mm}$ may impact on the point density calculation due to the high number of profiles. Table 6 has been collated to assist in understanding what may have contributed to MIMIC's calculation errors. Each of the input values was incremented individually until the predicted and measured values match. 
By examining this table, it is clear that even small discrepancies in specifying input values, measuring target orientation or system settings could account for those errors. Errors could not be quantified for the point spacing tests because an error of $2 \mathrm{~mm}$ at $5 \mathrm{~m}$ range is more significant than an error of $2 \mathrm{~mm}$ at $20 \mathrm{~m}$ range to the target.

Table 5. Error summary of point and profile tests using constructed CAD models and point cloud data from two real world MMSs.

\begin{tabular}{ccccc}
\hline Test & Output & Min $(\mathbf{m})$ & Mean $(\mathbf{m})$ & Max $(\mathbf{m})$ \\
\hline Constructed & $d_{\mathrm{PrSH}}$ & 0.000 & 0.000 & 0.000 \\
Constructed & $d_{\mathrm{PrSV}}$ & 0.000 & 0.000 & 0.000 \\
Constructed & $d_{\mathrm{PS}}$ & 0.000 & 0.000 & 0.000 \\
Real World & $d_{\mathrm{PrSH}}$ & 0.000 & 0.001 & 0.002 \\
Real World & $d_{\mathrm{PrSV}}$ & 0.000 & 0.001 & 0.002 \\
Real World & $d_{\mathrm{PS}}$ & 0.000 & 0.001 & 0.002 \\
\hline
\end{tabular}

Table 6. Assessment of errors for the horizontal and vertical profile spacing tests by quantifying the uncertainties in any of the input parameters that might account for errors in the real world tests.

\begin{tabular}{cccccccc}
\hline Surface & Err $(\mathbf{m})$ & $\boldsymbol{\alpha}_{\text {scan }}$ & $\boldsymbol{\gamma}_{\text {scan }}$ & $\boldsymbol{\alpha}_{\text {targ }}$ & $\boldsymbol{\beta}_{\text {targ }}$ & $\boldsymbol{M}_{\boldsymbol{f}}$ & Velocity $(\mathbf{m} / \mathbf{s})$ \\
\hline$d_{\mathrm{Pr} \mathrm{SH}}$ & 0.002 & $1.50^{\circ}$ & $\mathrm{n} / \mathrm{a}$ & $1.93^{\circ}$ & $\mathrm{n} / \mathrm{a}$ & $4.00 \mathrm{~Hz}$ & 0.200 \\
$d_{\mathrm{Pr} \mathrm{SV}}$ & 0.002 & $1.00^{\circ}$ & $0.5^{\circ}$ & $\mathrm{n} / \mathrm{a}$ & $1^{\circ}$ & $1.50 \mathrm{~Hz}$ & 0.130 \\
\hline
\end{tabular}

The versatility and accuracy of the system highlights some of the strengths of the method presented in this paper over existing methods [12,20,22,26-32]. MIMIC's geometric formulae incorporate scanner and target orientation, target and scanner height and all scanner settings. Carrying out a MMS survey and manually interrogating the data is a time consuming process. Additionally, with the manual approach, there is no complete understanding of what factors contribute to point and profile information. Measurements are required for every relevant MMS configuration and target type. To standardise this process, the tests must be performed on the same test-route. Without a powerful workstation, simulators can be slow, although work is ongoing to improve the speed of these systems [40]. Once the simulation is finished, measuring point distribution is a manual process and must be repeated for every area of interest. Simulators are optimal when modelling point distribution for large areas whereas the focus of this paper is on smaller objects, such as individual buildings or roadside infrastructure.

\section{Conclusions/Outlook}

This paper presented the geometric formulae required for calculating the profile spacing and the point spacing on angled targets. The profile spacing and point spacing calculations were validated in a series of tests using constructed CAD models as a control dataset and point cloud data from two MMSs to assess MIMIC's performance with real world data. The first output value from MIMIC was the profile spacing. MIMIC exhibited no error in the CAD tests, thus mathematically validating the profile spacing calculations. The results from the point cloud tests were also promising, with all errors below $2 \mathrm{~mm}$. The highest mean error for the profile spacing tests was $0.001 \mathrm{~m}$. An error of $1 \mathrm{~mm}$ is 
acceptable. The second output value from MIMIC was the point spacing. The control tests using the constructed 3D models once again returned no error, validating MIMIC's point spacing calculations mathematically. The highest mean error for the point spacing tests was $0.001 \mathrm{~m}$, which was encountered in the point cloud tests. Again, an error of $1 \mathrm{~mm}$ is acceptable. The main contribution of this paper is a method for calculating point and profile information for different MMS configurations on angled surfaces. It provides a valuable tool for assessing the performance of MMSs, designing survey specifications or tailoring automated algorithms to likely real world scenarios.

\section{Acknowledgments}

Research presented in this paper was funded by the Irish Research Council (IRC) and the Enterprise Partner, Pavement Management Services Ltd. (Galway, Ireland), by the NRA research fellowship program, ERA-NET SR01 projects and by a Strategic Research Cluster grant (07/SRC/I1168) from Science Foundation Ireland under the National Development Plan. The authors would also like to thank Optech Inc. for supplying additional test data.

\section{Author Contributions}

MIMIC was developed by Conor Cahalane, Conor P. McElhinney and Paul Lewis collaborated with Conor Cahalane in designing the procedures for experimental validation. Tim McCarthy designed and developed the XP1, used in validation, and provided technical advice at all stages. All authors contributed equally in preparing this manuscript.

\section{Conflicts of Interest}

The authors declare no conflict of interest.

\section{References}

1. McElhinney, C.P.; Kumar, P.; Cahalane, C.; McCarthy, T. Initial results from European Road Safety Inspection (EURSI) mobile mapping project. In Proceedings of the ISPRS Commission V Technical Symposium, Newcastle, UK, 21-24 June 2010.

2. Becker, S.; Haala, N. Grammar supported facade reconstruction from mobile LiDAR mapping. Int. Arch. Photogramm. Remote Sens. 2009, 38, 229-234.

3. Hammoudi, K.; Dornaika, F.; Paparoditis, N. Extracting building footprints from 3D point clouds using terrestrial laser scanning at street level. Int. Arch. Photogramm. Remote Sens. 2009, 38, $65-70$.

4. Kumar, P.; McCarthy, T.; McElhinney, C.P. Automated road extraction from terrestrial based mobile laser scanning system using the gvf snake model. In Proceedings of the European Laser Mapping Forum (ELMF 2010), The Hague, The Netherlands, 30-31 November 2010.

5. Kumar, P.; McElhinney, C.P.; McCarthy, T. Utilizing terrestrial mobile laser scanning data attributes for road edge extraction with the GVF snake model. In Proceedings of the MMT11, the 7th International Symposium on Mobile Mapping Technology, Krakaw, Poland, 13-16 June 2011. 
6. Pu, S.; Vosselman, G. Extracting windows from terrestrial laser scanning. Int. Arch. Photogramm. Remote Sens. Spat. Inform. Sci. 2007, 36, 320-325.

7. Pu, S.; Rutzinger, M.; Vosselman, G.; Elberink, S. Recognizing basic structures from mobile laser scanning data for road inventory studies. ISPRS J. Photogramm. 2011, 66, S28-S39.

8. Brenner, C. Global Localization of Vehicles Using Local Pole Patterns. In Pattern Recognition; Denzler, J., Notni, G., Se, H., Eds.; Springer-Berlin: Heidelberg, Germany, 2009; Lecture Notes in Computer Science Volume 5748, pp. 61-70.

9. Kukko, A.; Jaakola, A.; Lehtomäki, M.; Kaartinen, H.; Chen, Y. Mobile mapping system and computing methods for modelling of road environment. In Proceedings of the IEEE Joint Urban Remote Sensing Event, Shanghai, China, 20-22 May 2009.

10. Lehtomäki, M.; Jaakkola, A.; Hyyppä, J.; Kukko, A.; Kaartinen, H. Detection of vertical pole-like objects in a road environment using vehicle-based laser scanning data. Remote Sens. 2010, 2, 331-336.

11. Kaartinen, H.; Hyyppä, J.; Gulch, E.; Vosselman, G.; Hyyppä, H.; Matikainen, L.; Hofmann, A.D.; Mäder, U.; Persson, Å.; Söderman, U.; et al. Accuracy of 3D city models: EuroSDR comparison. Int. Arch. Photogramm. Remote Sens. Spat. Inform. Sci. 2005, 36, 227-232.

12. Yoo, H.; Goulette, F.; Senpauroca, J.; Lepere, G. Simulation based comparative analysis for the design of laser terrestrial mobile mapping. In Proceedings of the 6th International Symposium on Mobile Mapping Technology, Sao Paolo, Brazil, 21-24 July 2009; pp. 839-854.

13. Lari, Z.; Habib, A. Alternative methodologies for the estimation of local point density index: Moving towards adaptive LiDAR data processing. Int. Arch. Photogramm. Remote Sens. Spat. Inform. Sci. 2012, 39, 127-132.

14. Florida Department of Transport. Terrestrial Mobile LiDAR Surveying and Mapping Guidelines. Available online: http://www.dot.state.fl.us/surveyingandmapping/regulations.shtm (accessed on 5 October 2012).

15. Yen, W.; Ravani, B.; Lasky, T. LiDAR for Data Effiency. Available online: http://www.wsdot.wa.gov/Research/Reports/700/778.1.htm (accessed on 5 October 2011).

16. Olsen, M.J.; Roe, G.; Glennie, C.; Persi, F.; Reedy, M.; Hurwitz, D.; Williams, K.; Tuss, H.; Squellati, A.; Knodler, M. NCHRP 15-44 Guidelines for the Use of Mobile LiDAR in Transportation Applications, 2013. Available online: http://apps.trb.org/cmsfeed/ TRBNetProjectDisplay.asp?ProjectID=2972 (accessed on 3 April 2013).

17. Gonzalez-Jorge, H.; Riveiro, B.; Armesto, J.; Arias, P. Standard artifact for the geometric verification of terrestrial laser scanning systems. Opt. Laser Tech. 2011, 43, 1249-1256.

18. Lichti, D. A resolution measure for terrestrial laser scanners. Int. Arch. Photogramm. Remote Sens. Spat. Inform. Sci. 2004, 34, 216-221.

19. Lichti, D.; Jamtsho, S. Angular resolution of terrestrial laser scanners. Photogramm. Rec. 2006, 21, 141-160.

20. Hesse, C.; Kutterer, H. A mobile mapping system using kinematic terrestrial laser scanning (KTLS) for image acquisition. In Proceedings of the 8th Conference on Optical 3-D Measurement Techniques, Zurich, Switzerland, 9-12 July 2007; Grun, A., Kahmen, H., Eds.; pp. 134-141.

21. Glennie, C. A kinematic terrestrial LIDAR scanning system. Transp. Res. Board Natl. Acad. 2008, 281, 1-12. 
22. Kukko, A.; Andrei, C.O.; Salminen, V.M.; Kaartinen, H.; Chen, Y.; Rönnholm, P.; Hyyppä, H.; Hyyppä, J.; Chen, R.; Haggrén, H.; et al. Road environment mapping system of the Finnish Geodetic Institute-FGI Roamer. Int. Arch. Photogramm. Remote Sens. 2007, 36, 241-247.

23. Optech. Optech Lynx M1 and V200 System Specifications. Available online: http://www.optech.ca/lynx.htm (accessed on 9 October 2012).

24. Trimble. Trimble MX8 Mobile Mapping System. Available online: http://www.trimble.com/ geospatial/Trimble-MX8.aspx?dtID=overview (accessed on 9 October 2012).

25. 3D Laser Mapping, StreetMapper Technical Report. Available online: http://www.3dlasermapping. com/downloads/brochures/StreetMapperBrochure_v2.pdf (accessed on 5 October 2012).

26. Riegl. Dual Scanner Data sheet Riegl VMX-450. Available online: http://www.riegl.com/nc/products/ mobile-scanning/produktdetail/product/scannersystem/10/ (accessed on 9 October 2012).

27. Cahalane, C.; McCarthy, T.; McElhinney, C.P. Mimic: Mobile mapping point density calculator. In Proceedings of the 3rd International Conference on Computing for Geospatial Research and Applications (COM.Geo '12), Washington, DC, USA, 1-3 July 2012; pp. 15:1-15:9.

28. Riegl. RiACQUIRE Software Datasheet. Available online: http://products.rieglusa.com/item/ software-packages/riacquire-data-acquisition-software/item-1011 (accessed on 11 October 2012).

29. Hofmann, S.; Brenner, C. Quality assessment of automatically generated feature maps for future driver assistance systems. In Proceedings of the 17th ACM SIGSPATIAL International Conference on Advances in Geographic Information Systems (GIS '09), New York, NY, USA, 4-6 November 2009; pp. 500-503.

30. Puente, I.; Gonzalez-Jorge, H.; Martinez-Sanchez, J.; Arias, P. Review of mobile mapping and surveying technologies. Measurement 2013, 46, 2128-2144.

31. Lohani, B.; Mishra, R. Generating LiDAR data in laboratory: LiDAR simulator. Int. Arch. Photogramm. Remote Sens. 2007, 52, 12-14.

32. Kukko, A.; Hyyppä, J. Small-footprint laser scanning simulator for system validation, error assessment and algorithm development. Photogramm. Eng. Remote Sens. 2009, 75, 1177-1189.

33. Cahalane, C.; McElhinney, C.P.; McCarthy, T. Mobile mapping system performance: An analysis of the effect of laser scanner configuration and vehicle velocity on scan profiles. In Proceedings of the European laser Mapping Forum (ELMF 2010), The Hague, The Netherlands, 30-31 November 2010.

34. Cahalane, C.; McElhinney, C.P.; McCarthy, T. Calculating the Effect of Dual-Axis Scanner Rotations and Surface Orientation on Scan Profiles. In Proceedings of the 7th International Symposium on Mobile Mapping Technology (MMT11), Krakaw, Poland, 13-16 June 2011.

35. Cahalane, C.; McCarthy, T.; McElhinney, C.P. Mobile Mapping System Performance: An initial investigation into the effect of vehicle speed on laser scan lines. In Proceedings of the Remote Sensing \& Photogrammety Society Annual Conference -'From the Sea-Bed to the Cloudtops', Cork, Ireland, 1-3 September 2010.

36. Lewis, P.; McElhinney, C.P.; Schon, B.; McCarthy, T. Mobile mapping system LiDAR data framework. Int. Arch. Photogramm. Remote Sens. Spat. Inform. Sci. 2010, 38, 135-138. 
37. Lewis, P.; McElhinney, C.P.; McCarthy, T. Lidar data management pipeline; from spatial database population to web-application visualization. In Proceedings of the 3rd International Conference on Computing for Geospatial Research and Applications (COM.Geo '12), Washington, DC, USA, 1-3 July 2012; pp. 16:1-16:10.

38. McElhinney, C.P.; Lewis, P.; McCarthy, T. Mobile terrestrial LiDAR data-sets in a spatial database framework. In Proceedings of the MMT11, the 7th International Symposium on Mobile Mapping Technology, Krakaw, Poland, 13-16 June 2011.

39. Terrasolid. TerraScan User Guide. Available online: http://www.terrasolid./en/products/terrascan (accessed on 11 October 2012).

40. Kim, S.; Hwang, S.; Son, M.; LEE, I. Comprehensive high-speed simulation software for ladar systems. In Proceedings of the Earth Resources and Environmental Remote Sensing/GIS Applications, Prague, Czech Republic, 19 September 2011; doi:10.1117/12.898401.

(C) 2014 by the authors; licensee MDPI, Basel, Switzerland. This article is an open access article distributed under the terms and conditions of the Creative Commons Attribution license (http://creativecommons.org/licenses/by/3.0/). 